\title{
Endoscopic treatment of duodenal varices with cyanoacrylate
}

\author{
Ana Mora-Soler ${ }^{1}$, Antonio Velasco-Guardado ${ }^{1}$, Rosa Acosta-Materán ${ }^{1}$, Josué Umaña-Mejía ${ }^{1}$, Yuliana \\ Jamanca-Poma $^{1}$, Renzo Calderón-Begazo ${ }^{1}$, Jesús Legido-Gil ${ }^{2}$, Alberto Álvarez-Delgado ${ }^{1}$ and Antonio \\ Rodríguez-Pérez ${ }^{1}$
}

\section{${ }^{1}$ Department of Digestive Diseases. Hospital Universitario de Salamanca. Salamanca, Spain. ${ }^{2}$ Department of Digestive Diseases. Hospital General de Segovia. Segovia, Spain}

\begin{abstract}
Background: the duodenum is the most common location for ectopic varices. Bleeding is rare, but when it appears, it is massive and difficult to control.

Material and methods: retrospective description of five clinical cases of digestive bleeding secondary to duodenal varices that we observed between the years 2011 and 2012, together with their clinical characteristics, endoscopic diagnosis, endoscopic treatment with cyanoacrylate injection and the posterior follow-up and assessment of new bleeding. Results: all five patients were treated with an endoscopic cyanoacrylate injection and two of the patients experienced a digestive rebleeding. Three of the patients died during the follow-up period, only one due to cause digestive bleeding.

Conclusion: in conclusion we can state that endoscopic treatment of duodenal varices with cyanoacrylate is technically possible, and it permits us to control the first bleeding before doing other definitive treatments, if the patient condition allows it.
\end{abstract}

Key words: Duodenal varix. Digestive bleeding. Biological adhesive. Cyanoacrylate.

\section{INTRODUCTION}

Gastroesophageal varices are the most common complication in patients with portal hypertension. We define ectopic varices as those varices, which are not located in the gastroesophageal area. They are mainly located on the duodenum, jejunum, ileum, colon, rectum, and enterostomy stoma $(1,2)$. Ectopic varices present a bleeding prevalence of $2-5 \%$ (3), but it grows up to $17 \%$ when they are

Received: 09-04-2013

Accepted: 24-07-2013

Correspondence: Antonio Velasco Guardado. Department of Digestive Diseases. Hospital Universitario de Salamanca. Paseo San Vicente, 55-182. 37007 Salamanca, Spain

e-mail: antoniovelascog@usal.es located in the duodenum (2-4). Although it is relatively rare, the hemorrhage is usually massive and important, with a mortality rate of around $40 \%$ in its first episode $(1,4)$. The management of hemorrhage with a duodenal origin is a difficult task, not only due to the inaccessibility and difficult implementation of the treatment, but also because most of the studies include small case series with no randomized therapeutic essays that prove which therapy is the best one with enough evidence (5). Management options include medical treatment (3), endoscopic treatment $(6,7)$ as well as radiological (8) and surgical procedures $(4,5)$.

Duodenal varices are more common in patients with intrahepatic portal hypertension. Regarding its causes, liver cirrhosis is responsible for $30 \%$ of the cases. There are other causes, such as idiopathic portal hypertension or an extra-hepatic obstruction of the portal vein (1).

We describe retrospectively 5 cases of gastrointestinal bleeding from duodenal varices treated with cyanoacrylate.

\section{CASE REPORTS}

The clinical and endoscopic characteristics of the patients are shown in table I.

\section{Case report 1}

The patient is a 55-year-old woman with a personal history of hepatic echinococcosis operated in 2009, with portal hypertension secondary to portal cavernoma with

Mora-Soler A, Velasco-Guardado A, Acosta-Materán R, UmañaMejía R, Jamanca-Poma Y, Calderón-Begazo R, Legido-Gil R, Álvarez-Delgado A, Rodríguez-Pérez A. Endoscopic treatment of duodenal varices with cyanoacrylate. Rev Esp Enferm Dig 2013;105:629-633. 
no associated liver cirrhosis. She presented grade III-IV esophageal varices with no previous record of digestive bleeding. The patient was admitted with an acute episode of hematemesis and melenic stools of 15 days of evolution. An emergency gastroscopy was performed, revealing 4 large varicose cords from the middle esophageal third to the subcardial region, with red signs in its wall, although with no evidence of active bleeding. Nine ligation bands were inserted without immediate complications. The stomach and the duodenum did not present potentially bleeding lesions or red blood cell remains. Afterwards, the patient presented several episodes of melena with secondary anemization and the need for a transfusion of 22 red cell units. Two attempts were made to control the bleeding endoscopically, and they did not reveal hemorrhage in the crust of the previous bands or in any other gastrointestinal area. In a computed tomography (CT) done, there were esophageal varices secondary to chronic thrombosis of the spleno-portal axis, splenomegaly and ascites without identifying active bleeding. In a magnetic resonance (MR) was observed liver hydatid already described in previous studies, cavernomatosis associated with portal vein thrombosis and splenomegaly. In view of this fact, we decided to perform abdominal arteriography selective of celiac trunk and superior mesenteric artery without identifying active bleeding points or vascular malformations. In the absence of findings, we decided to perform an enteroscopy. In the superior duodenal flexure, we observed a conglomerate of submucosal varices that was confirmed with a radial echoendoscopy. We injected 3 milliliters $(\mathrm{ml})$ of adhesive (cyanoacrylate) on the duodenal varices with no immediate complications. After 48 hours with no recurrence of bleeding, the patient presented a high-volume hematemesis that caused a bronchoaspiration and a secondary cardiopulmonary arrest, in spite of the resuscitation procedures that were applied.

\section{Case report 2}

The patient is a 54-year-old man, hypertense, with diabetes and alcoholic liver cirrhosis Child C stage with portal hypertension. The patient had esophageal varices grade II and he had not been previously admitted with digestive bleeding. He was admitted with an episode of hematemesis and rectorrhagia. An emergency gastroscopy was performed and it revealed three small-medium varicose cords with red signs on the wall that involved the lower third of the esophagus, with no evidence of active bleeding at the exploration. Two ligation bands were inserted. An abdominal ultrasound revealed signs of chronic diffuse liver disease with signs of portal hypertension, reversed portal flow and ascites. After a new episode of melenic stools we performed an endoscopy that revealed three small varicose cords with 2 crusts on the distal third and a slight edema. The stomach presented red blood on the greater curvature. The duodenum presented a duodenal varix on the second portion with fibrin plug. We injected $1 \mathrm{ml}$ of cyanoacrylate and $4 \mathrm{ml}$ of aethoxysklerol (dilution $1 \%$ ). After the endoscopic treatment, the patient did not present any new episodes of apparent bleeding or anemization, and he was subsequently discharged. After 13 months of monitorization, the patient has not required new admissions caused by upper digestive bleeding secondary to bleeding caused by duodenal varices.

\section{Case report 3}

The patient is a 69-year-old man with a personal history of alcoholic liver cirrhosis with Child B stage and portal hypertension. He is admitted with a diagnosis of hepatic encephalopathy grade I-II. Twenty-four hours after admission, the condition of the encephalopathy worsened to grade III-IV and melenic stools appeared. An emergency gastros-

Table I. Clinic and endoscopic characteristics of the cases with duodenal varices bleeding

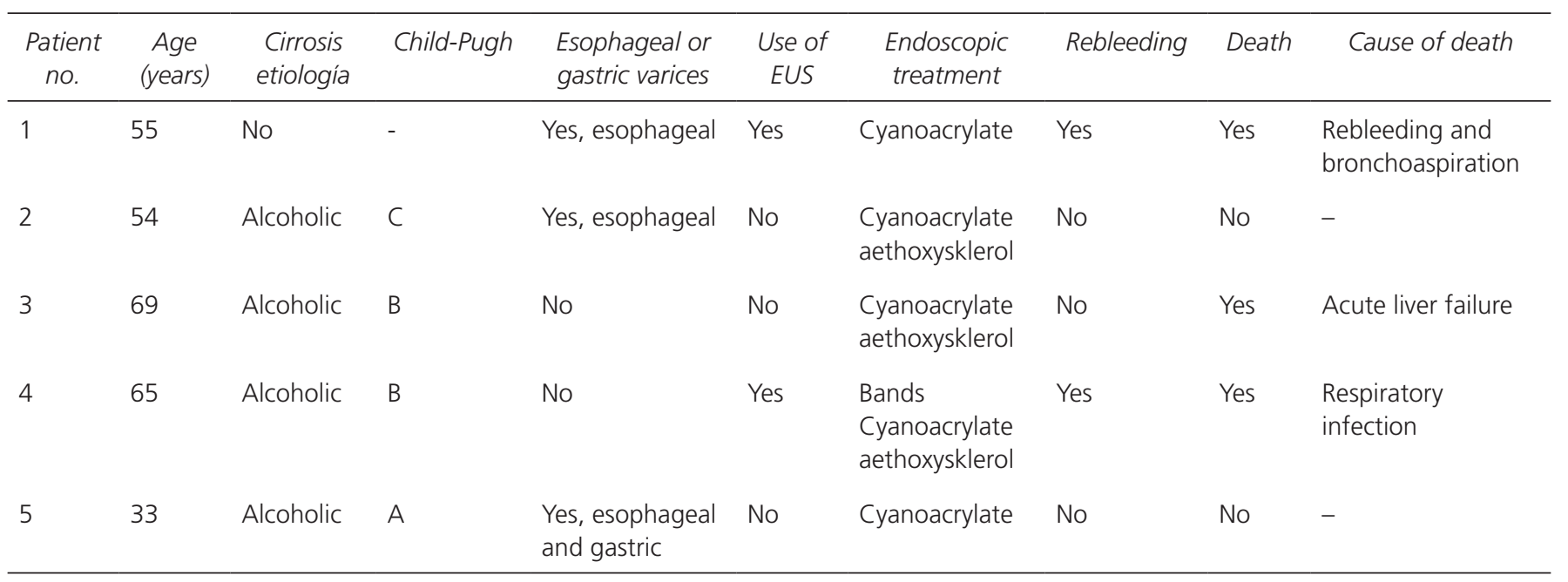


copy did not reveal signs of active bleeding, and there was no evidence of esophageal varices. An abdomino-pelvic CT showed signs of liver cirrhosis with portal hypertension, ascites, and evidence of increased caliber peripheral vessel that supplies the region of the cecum. After multiple episodes of gastrointestinal bleeding a colonoscopy was made observing traces of blood from small intestine. Because of that, an enteroscopy was performed, and it did not reveal varices or red cell remains in the esophagus or stomach. The second portion of the duodenum showed several varicose-like projections spurting blood in one of them. After sclerotizing it with aethoxysklerol (dilution $1 \%$ ), the bleeding increased, and we decided to apply sclerotherapy with $1 \mathrm{ml}$ cyanoacrylate in the two main varices, which stopped the bleeding once that the sclerotherapy needle was removed. After the enteroscopy, the patient presented a progressive decrease of his level of consciousness; as well as a deterioration of the kidney, liver, heart and neurological functions. Therapeutic effort limitation was applied and the patient died as a consequence of a liver failure.

\section{Case report 4}

The patient is a 65-year-old woman with a history of alcoholic liver cirrhosis and Child B stage, with no signs of portal hypertension. She was admitted with a diagnosis of hepatic encephalopathy III-IV. A few hours after admission, she presented melenic stools and hematochezia. An emergency gastroscopy was performed. It did not reveal esophageal varices or alterations in the stomach. The second duodenal portion revealed a thick duodenal varix with active spurting of blood. We applied sclerotherapy with $6 \mathrm{ml}$ of aethoxysklerol (dilution $1 \%$ ) and two bands on the varix which stopped the bleeding. Twenty-four hours after the endoscopic treatment, the patient presented a new episode of bleeding with $\mathrm{Hb}$ values of $5 \mathrm{~g} / \mathrm{dl}$. An emergency gastroscopy showed a deep ulcer with adherent clot and active oozing bleeding. The radial echoendoscopy showed a clear duodenal varix next to the ulcerated area. Sclerotherapy was administered with $1 \mathrm{ml}$ cyanoacrylate on the varicose area. The next days the patient presented new episodes of mild bleeding with progressive secondary anemization but no need of new endoscopic studies. The following weeks there were multiple complications but no new episodes of digestive bleeding: spontaneous bacterial peritonitis, right middle cerebral artery ischemic stroke and pneumonia. The patient was disconnected from mechanical ventilation and she died after 90 days of admission.

\section{Case report 5}

The patient is a 33-year-old man with a personal record of splenic and mesenteric vein thrombosis and portal cavernomatosis in 2007, with data of a recently diagnosed alcoholic chronic hepatopathy, Child grade A. He did not present pre- vious admissions with hepatic decompensation or bleeding. He was admitted as an emergency with symptoms of melenic stools and abdominal pain of 4-5 days of evolution together with 2-3 episodes of hematemesis. An emergency endoscopy revealed small esophageal varices with no red signs, larger esophageal-gastric varices type I, together with apparently large gastric varices type II (with no connection with the esophageal varices, and located away from the fundus) with stigmata of active bleeding. We injected $8 \mathrm{ml}$ of aethoxysklerol $1 \%$ on gastric varices. An abdominal ultrasound showed data compatible with chronic liver disease with portal vein intrahepatic filiform and signs of portal cavernous and splenomegaly. In abdominal CT was observed portal vein thrombosis and cavernous, partial thrombosis splenic vein, collateral circulation in splenic hilum, perigastric and mesenteric. Few days after, the patient presented a new episode of hematemesis associated with dizziness, excessive sweating and hypotension $(70 / 40 \mathrm{mmHg})$. An emergency gastroscopy revealed small esophageal and gastric varices that did not present signs of rupture. The duodenal bulb showed a large duodenal varix that spurted blood with erosion of its apex. Two $\mathrm{ml}$ of cyanoacrylate and $5 \mathrm{ml}$ of aethoxysklerol $1 \%$ were injected and the bleeding stopped (Fig. 1). No new exteriorization of bleeding was observed and the patient was discharged. After five months of monitoring, the patient has not presented new episodes of bleeding.

\section{DISCUSSION}

The best diagnostic method so far for locating the varices is digestive endoscopy, although it involves a limita-

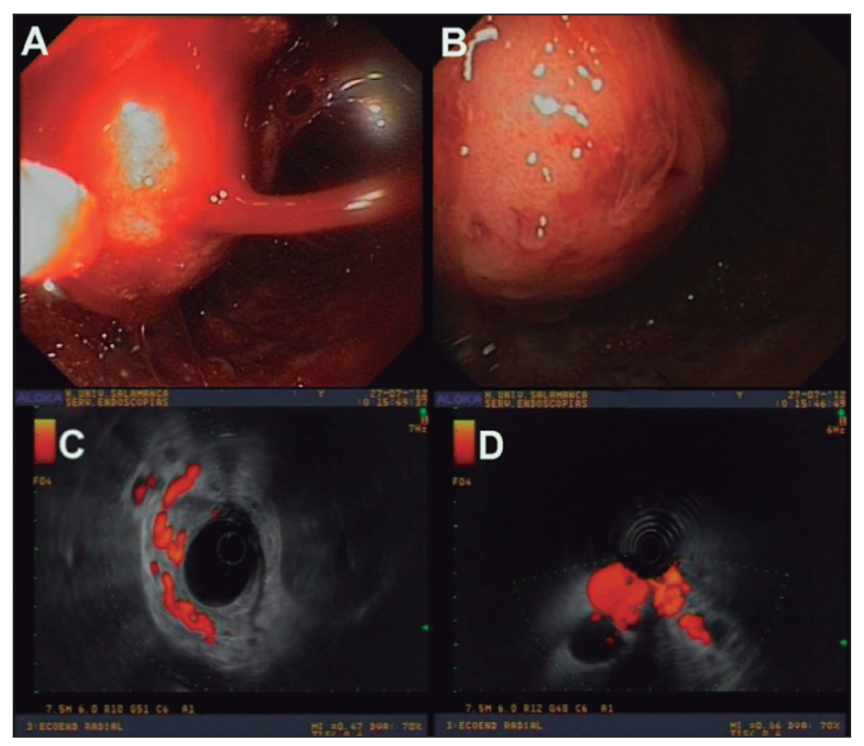

Fig. 1. A. Large duodenal varix with active bleeding in the duodenal bulb. B. Cessation of bleeding after the injection of cyanoacrylate and aethoxysklerol. C and D. EUS image showing the collateral circulation around the duodenum. 
tion on the localization of lesions in the submucosal and serous membranes (9). In these cases, endoscopic ultrasound (EUS) may help us identify submucosal lesions, so that we can observe the presence of a blood flow with Doppler-echo, as well as guide a proper treatment (4). We used the EUS in two patients. The exploration of the third portion of the duodenum is especially recommended with patients who present portal hypertension and a previous history of hemorrhage of digestive origin $(1,4)$. In our series, 4 out of the 5 patients presented at least one endoscopy previous to the diagnostic endoscopy, which did not reveal a duodenal pathology. In the last years, some endoscopic and radiological techniques have been developed for the treatment of ectopic varices in order to avoid surgery. Cyanoacrylate is a monomer-based tissue adhesive that instantly polymerizes and solidifies when it comes into contact with the hemorrhage. Liu et al. (4) developed a five-year retrospective study in 2009, including 14 patients with duodenal varices, and four of them had been treated with cyanoacrylate. In $100 \%$ of the cases treated with cyanoacrylate, the hemorrhage stopped immediately. Two deaths were registered, one of them due to liver failure 7 months after treatment and another one caused by multiple organ failure secondary to sepsis 24 after treatment. In the remaining patients no cases of rebleeding caused by duodenal varices was registered in the monitoring stages after 9 and 30 months. The study suggested that this treatment is safe and effective, both in acute hemorrhages and in deferred treatment. In our series, three patients died $(60 \%)$, although only one of the cases death secondary to bleeding (20\%), while the others two were caused by liver failure and respiratory infection. Treatment with cyanoacrylate controlled the bleeding in 4 out of 5 patients $(80 \%)$. In the cases described has been used a total dose of cyanoacrylate (not mixed) relatively low $(1-2 \mathrm{~mL}$ ) with respect to commonly used in gastric varices (10). If we consider that all cases are active bleeding, we inject the amount of glue needed to achieve hemostasis. Dulic et al. (6), in 2011, recorded a case of endoscopic ligation of a bleeding duodenal varix by stopping the control of the bleeding, with no immediate of delayed complications. We tried to do an endoscopic ligature in one case, but with poor results. The treatment options with interventional radiology are percutaneous transhepatic obliteration (PTO), transileocolic vein obliteration (TIO), balloon-occluded retrograde transvenous obliteration (B-RTO) and transjugular intrahepatic portosystemic shunt (TIPS) $(8,11)$. Treatment with TIPS reduces the incidence of the rebleeding, but it increases postoperative mortality and morbidity. Kochar et al. (6), in 2008, studied a large series of patients with bleeding caused by ectopic varices and treated with TIPS as an initial therapy. It was effective in $67 \%$ of 28 cases, and most of them did not require concomitant therapy. Twenty one percent of the patient showed rebleeding, in most cases secondary to shunt failure. The therapeutic approach with TIPS is considered to be a safe and effective option in the initial management of bleeding and in the prevention of recurrences, with a low and acceptable risk.

A study by Kakizaki et al. (12) in 2010 concluded that not all bleeding caused by duodenal varices requires an additional posterior treatment with surgery or interventional radiology, especially in those cases in which the endoscopic treatment has been effective. However, if we consider the high rate of rebleeding observed in our series (40\%), additional treatment, either surgery or radiotherapy, would be recommended.

In conclusion we can state that endoscopic treatment of duodenal varices with cyanoacrylate is technically possible, and it permit us to control the first bleeding before doing other definitive treatments, if the patient condition allows it. More studies are needed in order to establish the effectiveness and safety of this treatment in this atypical location of varices.

\section{REFERENCES}

1. Sato T, Akaike J, Toyota J, Karino Y, Ohmura T. Clinicopathological features and treatment of ectopic varices with portal hypertension. Int J Hepatol 2011;2011:960720.

2. Lebrec D, Benhamou JP. Ectopic varices in portal hypertension. Clin Gastroenterol 1985;14:105-21.

3. Norton ID, Andrews JC, Kamath PS. Management of ectopic varices. Hepatology 1998;28:1154-8.

4. Liu Y, Yang J, Wang J, Chai G, Sun G, Wang Z, et al. Clinical characteristics and endoscopic treatment with cyanoacrylate injection in patients with duodenal varices. Scand J Gastroenterol 2009;44:1012-6.

5. Kochar N, Tripathi D, Mcavoy NC, Ireland H, Redhead DN, Hayes PC. Bleeding ectopic varices in cirrhosis: The role of transyugular intrahepatic portosystemic stent shunts. Aliment Pharmacol Ther 2008;28:294-303.

6. Dulic M, Dulic-Lakovic E, Hellmich B, Blaha B, Gschwantler M. Successful treatment of a bleeding duodenal varix by endoscopic band ligation. J Gastrointestin Liver Dis 2011;20:234.

7. Ota K, Shirai Z, Masuzaki T, Tanaka K, Higashihara H, Okazaki M, et al. Endoscopic injection sclerotherapy with n-butyl-2-cyanoacrylate for ruptured duodenal varices. J Gastroenterol 1998;33:550-5.

8. Kakio T, Ito T, Sue K, Sakaguchi K, Shiota T, Oka T, et al. Hemostasis of gastric variceal hemorrhage by transileocoecal and transhepatic obliteration. Acta Med Okayama 1993;47:39-43.

9. Rana SS, Bhasin DK, Rao C, Singh K. Endoscopic ultrasound-guided treatment of bleeding duodenal varix. Indian J Gastroenterol 2011;30:280-1

10. Seewald S, Ang TL, Imazu H, Naga M, Omar S, Groth S, et al. A standardized injection technique and regimen ensures success and safety of $\mathrm{N}$-butyl-2-cyanoacrylate injection for the treatment of gastric fundal varices (with videos). Gastrointest Endosc 2008;68(3):447-54.

11. Jonnalagadda SS, Quiason S, Smith OJ. Successful therapy of bleeding duodenal varices by TIPS after failure of sclerotherapy. Am J Gastroenterol 1998;93:272-4.

12. Kakizaki S, Toyoda M, Ichikawa T, Sato K, Takagi H, Arai H, et al. Clinical characteristics and treatment for patients presenting with bleeding duodenal varices. Dig Endosc 2010;22:275-81. 